\title{
Effectiveness of a 5-year Community Oral Health Program for the Elderly in Korea
}

\author{
Eun-Joo Song and Soo-Jeong Hwang ${ }^{1 \dagger}$ \\ Daejeon Donggu Public Health Center, Daejeon 34691, \\ ${ }^{1}$ Department of Dental Hygiene, College of Medical Science, Konyang University, Daejeon 35365, Korea
}

\begin{abstract}
Korea has been running the community oral health program for the elderly, including topical fluoride application and scaling. The aim of this study was to compare the subjective and objective oral health status of 345 participants according to the number of participants in the program and of 37 participants before and after the 5 -year program. The survey consisted of an interview questionnaire and oral examinations. Analysis of variance was used to compare the variables of the 345 participants according to the numbers of participants. Paired t-test was used to compare the oral health statuses before and after the 5-year program in 37 subjects. There was no difference in subjective oral health status according to the number of participants in the oral health program in the elderly, including subjective health status, subjective oral health status, satisfaction with oral health, concern about oral health, need of dental treatment, oral pain, tooth sensitivity, subjective periodontal health, and subjective symptoms of periodontitis. The community periodontal index (CPI) of the 1 time participants was significantly higher than that of 3 times, 4 times or 5 times participants in the upper center, lower left, lower center, and lower right areas. There was a significant improvement in $\mathrm{CPI}$ from $2.59 \pm 1.14$ to $1.41 \pm 1.54(p<0.001)$ and positive oral behavioral change (daily tooth brushing frequency from $2.27 \pm 0.73$ to $2.54 \pm 0.90$ ) before and 5 years after the program. However, the program did not prevent tooth loss as the numbers of the remaining teeth significantly reduced from $23.77 \pm 1.84$ to $21.95 \pm 2.03$ over 5 years. We showed that running the community oral health program for the elderly for more than three years might have positive effects on the periodontal health of participants.
\end{abstract}

Key Words: Elderly, Korea, Oral health

\section{Introduction}

The proportion of older people continues to grow worldwide in developing countries ${ }^{1}$. According to Korean national demographic census in 2015, approximately $6,624,000$ people, which accounted for $13.1 \%$ of the population, were aged 65 years and over. The aging population has outnumbered the youth in 2017, and it will exceed $40 \%$ of the total population. Korea has introduced the 2020 Oral Health Goals to improve the oral health of the elderly. This includes increasing the proportion of the elderly with 20 or more teeth by $59.0 \%$, increasing the number of remaining teeth by 20 , reducing the prevalence of subjective mastication discomfort by $48.0 \%$, and inc- reasing the proportion of the elderly who have annual oral health checkups by $36.0 \%^{2)}$.

Korea has two community-driven oral health programs including prevention and rehabilitation for the elderly. The denture delivery program for low-income groups, which started in 2002, was stopped in 2016 because the national health insurance did not cover partial and full dentures for the elderly over 65 years of age. The fluoride application-scaling program for the elderly has been delivered in some communities, which started as a demonstration project in 2009 with national funds. After the integrated health promotion program in 2013, it has been promoted as an optional program according to the condition of each community. 
Daejeon Donngu Public Health Center has run the oral health promotion program for the elderly since 2009 . About $3 \%$ of the population of the elderly in this area has participated in this project every year. The dental hygienists in charge of the program insisted that the project should be evaluated to measure improvements in oral health status of the participants in spite of the difficulty in its management. Oral examinations and a questionnaire for the participants were conducted to confirm the improvement of oral health status in the participants through the program in 2013. The aim of this study was to inspect the difference of oral health status according to the numbers of years of participation and change of oral health status through the 5-year community oral health program.

\section{Materials and Methods}

\section{Study design and sampling}

This cross-sectional study was based on a sample of participants in the oral health promotion program for the elderly in Donggu, Daejeon in 2013, followed by a retrospective cohort study using the data from the survey of oral health status in this area in $2009^{3)}$. Among 1,257 participants aged $\geq 65$ years in 2013 , 355 subjects gave their written consent to participate in this survey. We excluded 10 participants with multiple missing responses in the questionnaire. There were 127 persons who participated once and 217 persons who had participated more than once in the program. Thirty-seven subjects in this study participated in the 2009 Oral Health Survey. Among the subjects, $57.4 \%$ were women, and the average age was $74.46 \pm 4.81$ years. This study was approved by the institutional review board of Konyang University Hospital (KYUH 13-92).

\section{Data collection}

One dentist performed oral examination in the program. The dentist had no prior knowledge of the participants to exclude prejudice, and the oral examination was carried out according to the criteria of the World Health Organization. Community periodontal index (CPI) and the number of remaining teeth were evaluated.

The interview questionnaire comprised an assessment of subjective health status, subjective oral health status, satisfaction with oral health, concern about oral health, need of dental treatment, oral pain, tooth sensitivity, subjective periodontal health, subjective symptoms of periodontitis, and oral health behavior. Subjective health and subjective oral health were assessed using a 6-point Likert scale. Assessment of satisfaction with oral health, concern about oral health, need for dental treatment, and subjective periodontal health was performed using 5-point Likert scales. Oral pain and tooth sensitivity were assessed using a 4-point Likert scale. Oral health behavioral was assessed using a 100-point Thurstone scale. Subjective symptoms of periodontitis consisted of swollen gums, sore gums, receding gums, loose teeth, drifting teeth, and bad breath. The positive and negative symptoms were mixed to achieve good reliability. In the statistical analysis, the higher the score, the more acceptable the item.

\section{Oral health promotion program in elderly ${ }^{4)}$}

The oral health promotion program for the elderly in Donggu, Daejeon, is a program in which dental hygienists search for and visit target persons directly. The dental hygienists responsible for the program contacted the managers of community centers, welfare centers, nursing homes, and silver halls, among others. They visited the places where the managers agreed to participate in the program. The program consisted of an oral examination, scaling with an ultrasonic scaler, fluoride varnish application with 5\% sodium fluoride, denture cleaning, individual oral health education, and supply of toothbrush and denture cleaner. The participants could attend the program only once a year.

\section{Statistical analysis}

The purposes of this analysis were to compare the subjective and objective oral health statuses according to the number of individuals participating in the program and to investigate whether an improvement in oral health occurred through the program. Analysis of variance was used to compare the variables according to the number of individuals participating in it. Paired t-test was used to compare the oral health status between 2009 and 2013 in 37 participants because the data were normally distributed 


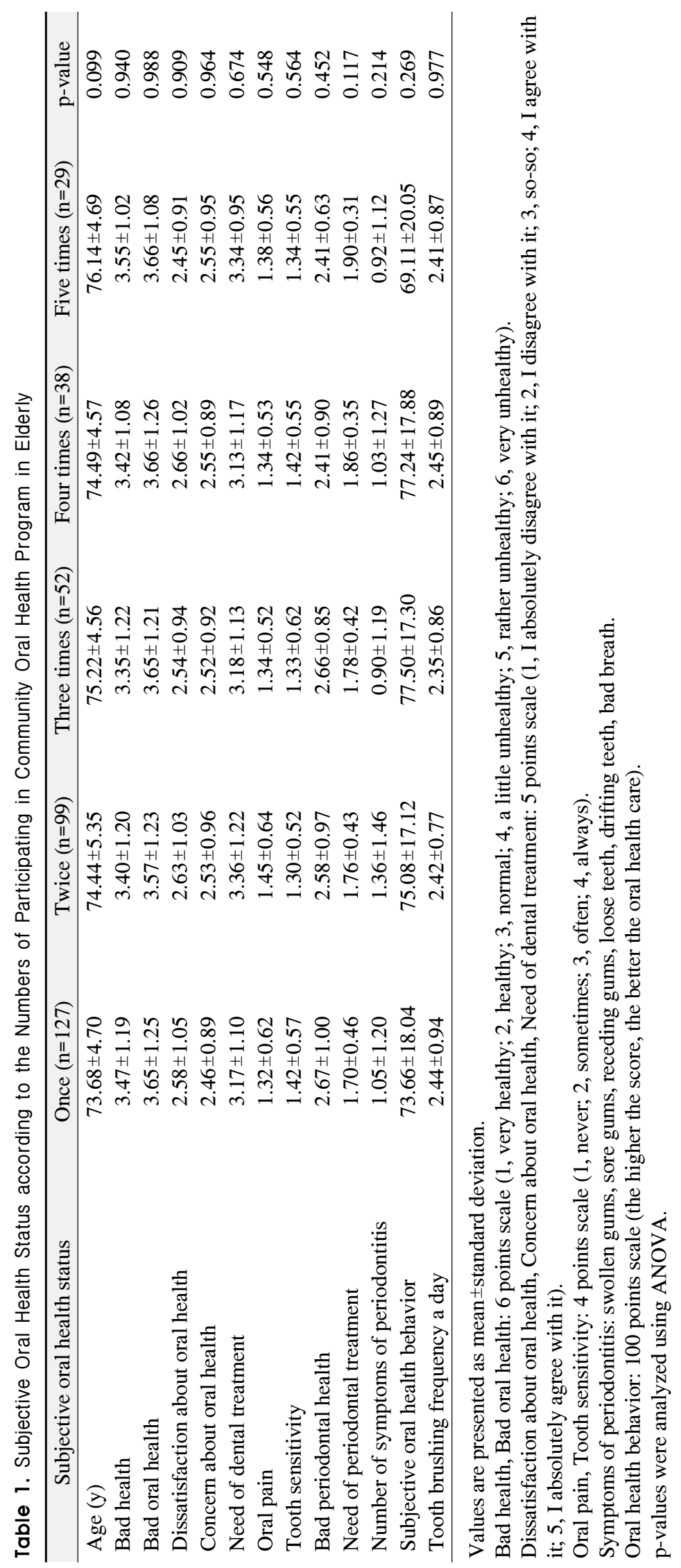


in spite of the small number of participants. The level of statistical significance was set at $\alpha=0.05$. All statistical analyses were performed with the IBM SPSS 20.0 (IBM Co., Armonk, NY, USA).

\section{Results}

There were no differences in subjective oral health status including subjective health status, subjective oral health status, satisfaction with oral health, concern about oral health, need of dental treatment, oral pain, tooth sensitivity, subjective periodontal health, and subjective symptoms of periodontitis according to the number of participants in the oral health program for the elderly (Table 1). There were some difference in CPI in the upper center, lower left, lower center, and lower right area

Table 2. Objective Oral Status according to the Numbers of Participating in Community Oral Health Program in Elderly

\begin{tabular}{lcccccc}
\hline Objective oral health status & Once $(\mathrm{n}=127)$ & Twice $(\mathrm{n}=99)$ & Three times $(\mathrm{n}=52)$ & Four times $(\mathrm{n}=38)$ & Five times $(\mathrm{n}=29)$ & $\mathrm{p}$-value \\
\hline CPI & $1.60 \pm 1.44$ & $1.36 \pm 1.53$ & $1.20 \pm 1.46$ & $1.26 \pm 1.52$ & $1.22 \pm 1.50$ & 0.416 \\
CPI-upper right & $0.57 \pm 0.21$ & $0.61 \pm 1.28$ & $0.63 \pm 1.21$ & $0.21 \pm 0.86$ & $0.62 \pm 1.32$ & 0.555 \\
CPI-upper center & $0.56 \pm 1.21^{\mathrm{a}}$ & $0.25 \pm 0.87^{\mathrm{a}, \mathrm{b}}$ & $0.26 \pm 0.96^{\mathrm{a}, \mathrm{b}}$ & $0.08 \pm 0.49^{\mathrm{b}}$ & $0.08 \pm 0.50^{\mathrm{b}}$ & 0.015 \\
CPI-upper left & $0.71 \pm 1.29$ & $0.51 \pm 1.18$ & $0.50 \pm 1.13$ & $0.56 \pm 1.24$ & $0.67 \pm 1.34$ & 0.803 \\
CPI-lower left & $1.02 \pm 1.34^{\mathrm{a}}$ & $0.71 \pm 1.29^{\mathrm{a}, \mathrm{b}}$ & $0.45 \pm 1.07^{\mathrm{a}, \mathrm{b}}$ & $0.16 \pm 0.64^{\mathrm{b}}$ & $0.50 \pm 1.03^{\mathrm{a}, \mathrm{b}}$ & 0.004 \\
CPI-lower center & $1.11 \pm 1.22^{\mathrm{a}}$ & $0.86 \pm 1.25^{\mathrm{a}, \mathrm{b}}$ & $0.45 \pm 0.90^{\mathrm{b}}$ & $0.63 \pm 1.02^{\mathrm{b}}$ & $0.50 \pm 0.95^{\mathrm{b}}$ & 0.004 \\
CPI-lower right & $1.01 \pm 1.30^{\mathrm{a}}$ & $0.64 \pm 1.30^{\mathrm{a}, \mathrm{b}}$ & $0.12 \pm 0.54^{\mathrm{b}}$ & $0.59 \pm 1.33^{\mathrm{a}, \mathrm{b}}$ & $0.54 \pm 1.06^{\mathrm{a}, \mathrm{b}}$ & 0.002 \\
Total remaining teeth & $19.68 \pm 8.05$ & $19.68 \pm 8.10$ & $19.54 \pm 7.97$ & $22.11 \pm 5.29$ & $21.52 \pm 8.22$ & 0.358 \\
Upper anterior teeth & $4.50 \pm 2.00$ & $4.71 \pm 2.04$ & $4.40 \pm 2.32$ & $5.03 \pm 1.35$ & $5.03 \pm 2.03$ & 0.405 \\
Upper posterior teeth & $5.17 \pm 2.90$ & $4.94 \pm 2.84$ & $4.69 \pm 2.94$ & $6.08 \pm 1.96$ & $5.59 \pm 2.88$ & 0.148 \\
Lower anterior teeth & $4.85 \pm 1.85$ & $4.87 \pm 1.96$ & $4.98 \pm 1.69$ & $5.45 \pm 1.22$ & $4.86 \pm 1.94$ & 0.474 \\
Lower posterior teeth & $5.17 \pm 2.64$ & $5.16 \pm 2.66$ & $5.46 \pm 1.22$ & $5.55 \pm 2.01$ & $6.03 \pm 2.50$ & 0.465 \\
\hline
\end{tabular}

Values are presented as mean \pm standard deviation.

CPI: community periodontal index ( 0 , healthy periodontal condition; 1 , dental plaque; 2 , calculus; 3 , shallow periodontal pocket; 4 , deep periodontal pocket).

${ }_{\mathrm{a}, \mathrm{b}}$ The same characters are not significant by Tukey post-hoc analysis; $\mathrm{p}$-values were analyzed using ANOVA.

Table 3. Change of Subjective Oral Health Status between Pre and Post 5 -Year Community Oral Health Program ( $n=37)$

\begin{tabular}{lccc}
\hline \multicolumn{1}{c}{ Subjective oral health status } & Before the program (2009 year) & After 5 years (2013 year) & p-value \\
\hline Bad health & $3.31 \pm 0.92$ & $3.56 \pm 1.13$ & 0.071 \\
Bad oral health & $3.08 \pm 0.81$ & $3.19 \pm 1.14$ & 0.524 \\
Dissatisfaction about oral health & $3.00 \pm 0.79$ & $3.25 \pm 0.97$ & 0.212 \\
Concern about oral health & $2.64 \pm 0.90$ & $2.64 \pm 0.96$ & 1.000 \\
Need of dental treatment & $2.50 \pm 0.88$ & $3.14 \pm 0.99$ & 0.004 \\
Oral pain & $1.42 \pm 0.55$ & $1.39 \pm 0.55$ & 0.800 \\
Tooth sensitivity & $1.58 \pm 0.60$ & $1.47 \pm 0.56$ & 0.291 \\
Bad periodontal health & $2.17 \pm 0.91$ & $2.53 \pm 0.70$ & 0.041 \\
Number of symptoms of periodontitis & $1.45 \pm 1.31$ & $0.97 \pm 1.17$ & 0.057 \\
Subjective oral health behavior & $65.81 \pm 17.74$ & $71.39 \pm 17.74$ & 0.209 \\
Tooth brushing frequency a day & $2.27 \pm 0.73$ & $2.54 \pm 0.90$ & 0.003 \\
\hline
\end{tabular}

Values are presented as mean \pm standard deviation.

Bad health, Bad oral health: 6 points scale (1, very healthy; 2, healthy; 3 , normal; 4 , a little unhealthy; 5 , rather unhealthy; 6 , very unhealthy).

Dissatisfaction about oral health, Concern about oral health, Need of dental treatment: 5 points scale (1, I absolutely disagree with it; 2 , I disagree with it; 3, so-so; 4, I agree with it; 5, I absolutely agree with it).

Oral pain, Tooth sensitivity: 4 points scale (1, never; 2 , sometimes; 3 , often; 4, always).

Symptoms of periodontitis: swollen gums, sore gums, receding gums, loose teeth, drifting teeth, bad breath.

Oral health behavior: 100 points scale (the higher the score, the better the oral health care).

p-values were analyzed using paired t-test. 
according to the number of participants (Table 2). The CPI of the 1 time participants was significantly higher than that of 3 times, 4 times or 5 times participants in the upper center, lower left, lower center, and lower right areas. There was no difference in the number of remaining teeth.

Regarding the difference in oral health status during the 5-year program, 37 subjects after the program thought they needed more dental treatment $(\mathrm{p}=0.004)$ and they had worse periodontal health $(p=0.041$; Table 3$)$. However, they brushed their teeth more often after the program $(p=0.003)$. They said their oral health behavior improved after the program, but there was no statistical difference. CPI in all areas was statistically improved after the 5-year program although the number of remaining teeth decreased significantly (Table 4).

\section{Discussion}

The oral health of the elderly is a cumulative consequence of dental caries and periodontal disease over a lifetime ${ }^{1)}$. There is a prejudice that oral disease prevention program for the elderly is less important and the oral health education for the elderly is less effective than that for other ages. However, Petersen and Yamamoto ${ }^{1)}$ found that older people did not hesitate to develop new oral health habits for improved health. Prayoonwong et al. ${ }^{5)}$ insisted the new community-based care models to enhance oral health care for the elderly are needed. The importance of oral health programs for the elderly should not be over looked because the elderly population and life expectancy is increasing. The Australian government has invested in improving the oral health of elderly people in $2010^{6}$, and the Korean government has driven the fluoride application-scaling program for the elderly since $2009^{4}$.

In Korean studies about the oral health of the elderly, regular dental visits had a significant impact on the oral health of the elderly ${ }^{7)}$, and the participants' satisfaction rate regarding their oral condition and the frequency of tooth brushing increased ${ }^{8)}$. Experience in oral health education had a positive impact on oral health knowledge, attitudes, and behavior, especially on periodic scaling ${ }^{9)}$. The group with more interest in oral health was likely to brush their teeth more often, and those with more frequent tooth brushing had higher oral health knowledge ${ }^{10)}$. Some studies showed oral health education for the elderly was also effective ${ }^{11)}$; it improved tooth brushing and flossing ability and reduced gingival bleeding ${ }^{12)}$. The participants' oral behavior in this study changed positively because the result showed that they brushed their teeth more often compared to before the program (Table 3). Besides, the objective oral health examinations showed an improvement in periodontal health indicators. CPI decreased by one

Table 4. Change of Objective Oral Health Status between Pre and Post 5-Year Community Oral Health Program ( $n=37$ )

\begin{tabular}{lcrr}
\hline Objective oral health status & Before the program (2009 year) & After 5 years (2013 year) & p-value \\
\hline CPI & $2.59 \pm 1.14$ & $1.41 \pm 1.54$ & $<0.001$ \\
CPI-upper right & $1.72 \pm 1.58$ & $0.55 \pm 1.24$ & 0.002 \\
CPI-upper center & $1.09 \pm 1.50$ & $0.00 \pm 0.00$ & $<0.001$ \\
CPI-upper left & $1.94 \pm 1.50$ & $0.76 \pm 1.37$ & 0.001 \\
CPI-lower left & $1.54 \pm 1.50$ & $0.46 \pm 1.09$ & $<0.001$ \\
CPI-lower center & $1.61 \pm 1.59$ & $0.64 \pm 1.22$ & 0.003 \\
CPI-lower right & $1.97 \pm 1.13$ & $0.64 \pm 1.10$ & $<0.001$ \\
Total remaining teeth & $23.77 \pm 1.84$ & $21.95 \pm 2.03$ & 0.047 \\
Upper anterior teeth & $5.21 \pm 1.54$ & $5.03 \pm 1.63$ & 0.181 \\
Upper posterior teeth & $6.26 \pm 2.42$ & $5.64 \pm 2.68$ & 0.004 \\
Lower anterior teeth & $5.54 \pm 1.43$ & $5.13 \pm 1.72$ & 0.031 \\
Lower posterior teeth & $6.76 \pm 1.97$ & $6.16 \pm 2.07$ & 0.003 \\
\hline
\end{tabular}

Values are presented as mean \pm standard deviation.

CPI: community periodontal index ( 0 , healthy periodontal condition; 1 , dental plaque; 2 , calculus; 3 , shallow periodontal pocket; 4 , deep periodontal pocket).

p-values were analyzed using paired t-test. 
point after the 5-year program (Table 4). Because those who participated more than 3 times showed significant improvement in CPI, we insist that the community oral health program is required for at least three years.

However, the participants in this program did not report a significant improvement in subjective oral health according to the number of years of participation (Table 1); they experienced symptoms of reduced periodontal health more, and they needed dental treatment more often after the program than before the program (Table 3 ). This program did not prevent tooth loss because 37 participants lost an average of 1.82 teeth over 5 years (Table 4 ). The negative view of their oral health might have led to their continued participation in the program. Komulainen et al. ${ }^{13)}$ reported that oral health intervention among the community-dwelling elderly reduced the incidence of oral diseases or symptoms to 0 in both the intervention and control groups at the 2-year follow-up; however, the difference between the groups was not significant. Gagliardi et al. ${ }^{14)}$ found that dental treatment intervention improved the quality of life regarding oral health; however, no improvement was seen in subjects with pain or discomfort. One study did not show significant relationships among objective oral examination, subjective oral health status, and requirement of dental treatment ${ }^{15)}$. Some previous studies have shown that the effects of oral health promotion programs for the elderly were not clear. There are many confounding variables to consider before concluding on the effectiveness of oral health program for the elderly because major oral diseases are cumulative diseases and there are the limitations on treating them through just prevention. Besides, we only offered oral health programs once a year, and there was no control over the participants' other behaviors. McGrath et al. ${ }^{16)}$ insisted that higher-quality research is required to provide more definite guidelines on oral health promotion practices for the elderly people.

The limitation of this study was the sampling bias because the research was designed using only the data from the community programs, without a control group. People who voluntarily participated in the program might have more interest in their oral health than general people. No reliability tests were performed on the oral examiner of this survey; however, she had been trained to measure reliability before the study. Nonetheless, this study could be valuable because the previous literature on the effects of the oral health promotion program for the elderly is scarce in Korea. The oral health promotion program in Korea might improve the periodontal health and oral health behavior in the elderly because this study showed there were significant improvements in CPI after the program. The participants had better oral health condition with more remaining teeth compared to the 2015 National Health Nutrition Survey ${ }^{17)}$, although they lost their teeth $1.82 \pm 3.12$ over 5 years. We have shown here that running the community oral health program for the elderly for more than three years might have positive effects on the periodontal health of participants.

\section{References}

1. Petersen PE, Yamamoto T: Improving the oral health of older people: the approach of the WHO global oral health programme. Community Dent Oral Epidemiol 33: 81-92, 2005.

2. Ministry of Health and Welfare: HP 2020, 2011-2020. Retrieved March 10, 2017, from http://www.mohw.go.kr/ front_new/jb/sjb030301vw.jsp?PAR_MENU_ID=03\&MEN U_ID=0319\&CONT_SEQ=257824\&page=1(2011, July 4).

3. Kim HY, Hwang SJ: Association of smoking, economic status and oral health in the elderly in Dong-gu, Daejeon. J Korean Acad Oral Health 35: 67-76, 2011.

4. Song EJ, Hwang SJ: Oral health promotion program for elderly in Dong-gu, Daejeon: a case report. J Dent Hyg Sci 16: 249-255, 2016.

5. Prayoonwong T, Wiwatkhunupakan T, Lasuka D, Srisilapanan P: Development of a community-based oral healthcare model for Thai dependent older people. Gerodontology 33: 545-553, 2016

6. Lewis A, Wallace J, Deutsch A, King P: Improving the oral health of frail and functionally dependent elderly. Aust Dent J 60 Suppl 1: 95-105, 2015.

7. Lee HO, Kim J: Effects of elders' oral health beliefs and oral health behaviors on their quality of life. J Dent Hyg Sci 8: 57-63, 2008.

8. Park MS, Choi MS: The efficiency on the oral health training of elderly adults at welfare institutions. J Dent Hyg Sci 8: 


$$
\text { 347-352, } 2008 .
$$

9. Jung JY, Han SJ: A study on oral health knowledge, behavior and education needs of the elderly. J Dent Hyg Sci 11: 513-520, 2011.

10. Lee YK, Han HJ: A study on oral health knowledge and behavior among the elderly. J Dent Hyg Sci 12: 189-195, 2012.

11. Cheung C, Tong M, Lum C: Effectiveness of an oral health care education programme delivered by elderly ambassadors: a quasi-randomised controlled trial. Asian J Gerontol Geriatr 1: 5-13, 2006.

12. Little SJ, Hollis JF, Stevens VJ, Mount K, Mullooly JP, Johnson BD: Effective group behavioral intervention for older periodontal patients. J Periodontal Res 32: 315-325, 1997.

13. Komulainen K, Ylöstalo P, Syrjälä AM, et al.: Oral health intervention among community-dwelling older people: a randomised 2-year intervention study. Gerodontology 32: 62-72, 2015.

14. Gagliardi DI, Slade GD, Sanders AE: Impact of dental care on oral health-related quality of life and treatment goals among elderly adults. Aust Dent J 53: 26-33, 2008.

15. Shin MS, Won YS, Gwon MY, Kim YS: A comparative study on DMFS, DMFT and FS-T indexes in the Korean elderly. J Dent Hyg Sci 10: 251-257, 2010.

16. McGrath C, Zhang W, Lo EC: A review of the effectiveness of oral health promotion activities among elderly people. Gerodontology 26: 85-96, 2009.

17. Korea Centers for Disease Control: 2015 National Health Nutrition Survey. Retrieved March 10, 2017, from http:// www.cdc.go.kr/CDC/contents/CdcKrContentView.jsp?cid= 60949\&menuIds=HOME001-MNU1130-MNU1639-MNU1 749-MNU1761(2016, December 21). 